\title{
White Matter Lesion Penumbra Shows Abnormalities on Structural and Physiologic MRIs in the Coronary Artery Risk Development in Young Adults Cohort
}

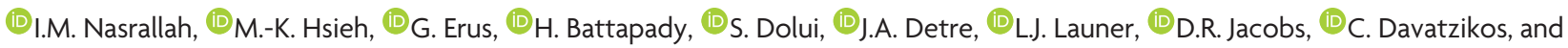
(1D.N. Bryan

\begin{abstract}
BACKGROUND AND PURPOSE: White matter lesions are 1 age-related manifestation of cerebrovascular disease, but subthreshold abnormalities have been identified in nonlesional WM. We hypothesized that structural and physiologic MR imaging findings of early cerebrovascular disease can be measured in middle-aged subjects in tissue adjacent to WM lesions, termed "penumbra."
\end{abstract}

MATERIALS AND METHODS: WM lesions were defined using automated segmentation in 463 subjects, $43-56$ years of age, from the Coronary Artery Risk Development in Young Adults (CARDIA) longitudinal observational cohort study. We described 0- to 2-mm and 2- to 4-mm-thick spatially defined penumbral WM tissue ROls as rings surrounding WM lesions. The remaining WM was defined as distant normal-appearing WM. Mean signal intensities were measured for FLAIR, T1-, and T2-weighted images, and from fractional anisotropy, mean diffusivity, CBF, and vascular reactivity maps. Group comparisons were made using Kruskal-Wallis and pair-wise $t$ tests.

RESULTS: Lesion volumes averaged $0.738 \pm 0.842 \mathrm{~cm}^{3}$ (range, $0.005-7.27 \mathrm{~cm}^{3}$ ). Mean signal intensity for FLAIR, T2, and mean diffusivity was increased, while $\Pi$, fractional anisotropy, and CBF were decreased in white matter lesions versus distant normal-appearing WM, with penumbral tissues showing graded intermediate values (corrected $P<.001$ for all group/parameter comparisons). Vascular reactivity was significantly elevated in white matter lesions and penumbral tissue compared with distant normal-appearing white matter (corrected $P \leq .001$ ).

CONCLUSIONS: Even in relatively healthy 43- to 56-year-old subjects with small white matter lesion burden, structural and functional MR imaging in penumbral tissue reveals significant signal abnormalities versus white matter lesions and other normal WM. Findings suggest that the onset of WM injury starts by middle age and involves substantially more tissue than evident from focal white matter lesions visualized on structural imaging.

ABBREVIATIONS: BMI = body mass index; BOLD = blood oxygen level-dependent; dNAWM = distant normal-appearing white matter; FA = fractional anisotropy; $\mathrm{MD}=$ mean diffusivity; $\mathrm{NAWM}=$ normal-appearing white matter; $\mathrm{RF}_{\text {score }}=$ risk factor score; $\mathrm{VR}=$ vascular reactivity; $\mathrm{WML}=$ white matter lesion

C

erebral white matter lesions (WMLs), or leukoaraiosis, are common age-related MR imaging findings but may be pres-
Received February 7, 2019; accepted after revision June 6.

From the Department of Radiology (I.M.N., R.N.B.), Center for Biomedical Image Computing and Analytics (I.M.N., M.-K.H., G.E., H.B., C.D.), and Department of Neurology (S.D., J.A.D.), University of Pennsylvania, Philadelphia, Pennsylvania; National Institute on Aging (L.J.L.), National Institutes of Health, Bethesda, Maryland; and Division of Epidemiology (D.R.J.), School of Public Health, University of Minnesota, Minneapolis, Minnesota.

The Coronary Artery Risk Development in Young Adults Study (CARDIA) is conducted and supported by the National Heart, Lung, and Blood Institute in collaboration with the University of Alabama at Birmingham (HHSN268201800005I, HHSN268201800007I), Northwestern University (HHSN268201800003I), University of Minnesota (HHSN268201800006I), and the Kaiser Foundation Research Institute (HHSN268201800004I). CARDIA is also partially supported by the Intramural Research Program of the National Institute on Aging and an intra-agency agreement between National Institute on Aging and National Heart, Lung, and Blood Institute (AG0005). This article has been reviewed by CARDIA for scientific content. Data processing and analysis was supported by NIH grants S10OD023495, P41 EB015893, P30 AG010124, and R01 NS111115. ent in younger individuals. ${ }^{1}$ WMLs are associated with cognitive decline, ${ }^{2-4}$ future infarction, depression, ${ }^{5}$ and poor clinical prognosis. ${ }^{6}$ WMLs commonly affect terminal vascular territories and their burden progresses ${ }^{7}$ by expansion of existing lesions and development of new lesions.

Pathologically, WMLs demonstrate capillary loss, arterial tortuosity, gliosis, ${ }^{8}$ demyelination, and ischemia. ${ }^{9}$ Imaging has shown physiologic abnormalities in WMLs, including decreased cerebral blood flow ${ }^{10,11}$ and vascular reactivity (VR), ${ }^{12}$ and in-

Paper previously presented, in part, at: American Society of Neuroradiology Annual Meeting and the Foundation of the ASNR Symposium, May 21-26, 2016; Washington DC.

Please address correspondence to llya M. Nasrallah, MD, PhD, Department of Radiology, University of Pennsylvania, 3400 Spruce St, Philadelphia, PA 19104; e-mail: ilya.nasrallah@pennmedicine.upenn.edu

- Indicates open access to non-subscribers at www.ajnr.org

三 Indicates article with supplemental online tables.

Indicates article with supplemental online photos.

http://dx.doi.org/10.3174/ajnr.A6119 
Table 1: Demographic and risk factor data of the subjects from CARDIA with MRI included in this study

\begin{tabular}{lcccc}
\hline & Total & KPDR & UMN & P Value \\
\hline No. & 463 & 213 & 250 & NA \\
Mean age (yr) & $50.5 \pm 3.4$ & $50.5 \pm 3.4$ & $50.5 \pm 3.3$ & .98 \\
Race & & & & \\
$\quad$ Black & $36 \%$ & $38 \%$ & $34 \%$ & .48 \\
$\quad$ White & $64 \%$ & $62 \%$ & $66 \%$ & \\
Sex & & & & \\
$\quad$ Male & $47 \%$ & $47 \%$ & $46 \%$ & .98 \\
$\quad$ Female & $53 \%$ & $53 \%$ & $54 \%$ & \\
Mean BMI & $28.3 \pm 5.3$ & $27.6 \pm 5.4$ & $28.9 \pm 5.2$ & .009 \\
Mean systolic/diastolic & $118 \pm 15 / 74 \pm 12$ & $118 \pm 14 / 74 \pm 11$ & $119 \pm 16 / 73 \pm 12$ & $.58 / .66$ \\
$\quad$ blood pressure (mm Hg) & & & & \\
Diabetic (\%) & $7.6 \%$ & $6.9 \%$ & $8.2 \%$ & .61 \\
Smoking history & & & & .005 \\
$\quad$ Never & $60 \%$ & $67 \%$ & $53 \%$ & \\
$\quad$ Former & $25 \%$ & $22 \%$ & $28 \%$ & \\
$\quad$ Current & $15 \%$ & $11 \%$ & $19 \%$ & .55 \\
Sedentary behavior & $19 \%$ & $18 \%$ & $20 \%$ & .38 \\
$\quad(>75$ th percentile) & & & $68 \%$ & \\
Hypercholesterolemia & $69 \%$ & $1.9 \pm 1.2$ & $2.2 \pm 1.3$ & .06 \\
Mean risk factor score & $2.1 \pm 1.3$ & & & \\
$\quad$ (range, 0-6) & & &
\end{tabular}

Note:-KPDR indicates Kaiser-Permanente Division of Research; UMN, University of Minnesota; NA, not applicable.

suggest that antihypertensive therapy and other therapies to control vascular risk factors may benefit brain health starting in middle age. We investigated tissue immediately surrounding WMLs, termed WML penumbra, which was likely exposed to similar vascular stressors, predicting that the imaging correlates of injury would be intermediate between WMLs and distant NAWM. We simultaneously characterized structural abnormalities, measures of white matter integrity, and vascular physiologic parameters to determine the characteristics of WML penumbral tissue using MR imaging.

\section{MATERIALS AND METHODS Study Sample}

The CARDIA study is a prospective, longitudinal cohort study evaluating the development of vascular risk factors in healthy young adults who provided in-

creased blood-brain barrier permeability ${ }^{13}$; other studies have established that age, hypertension, and smoking are important risk factors for WMLs. ${ }^{14}$ These observations lead to the hypothesis that cardiovascular risk factors result in chronic vascular impairment that causes tissue damage visualized as WMLs, though there is also evidence for progression through acute injury. ${ }^{15}$ Together, these injuries likely interfere with axonal function and connectivity, contributing to clinical deficits, including cognitive decline.

Many methods exist to automatically segment WMLs from MR imaging, usually relying on structural MR imaging signal intensity characteristics. Because WMLs are usually progressive, MR imaging techniques sensitive to early pathologic changes may identify at-risk tissue in normal-appearing white matter (NAWM). Indeed, MR imaging studies, mostly in elderly populations, ${ }^{7,16,17}$ have demonstrated abnormalities in several measurements of NAWM on conventional MR imaging. NAWM near WMLs is most likely to show abnormality. ${ }^{17}$ One large study evaluating signal abnormalities in NAWM found increased FLAIR signal, increased mean diffusivity (MD), and decreased fractional anisotropy (FA) in tissue that ultimately developed into WMLs, ${ }^{7}$ while a smaller study showed similar findings for low CBF. ${ }^{18}$ White matter integrity metrics from diffusion tensor imaging, like FA and MD, are also abnormal in NAWM in individuals with WMLs. ${ }^{19-21}$ However, many previous studies are limited by small sample sizes and ROI image-analysis techniques that do not broadly evaluate WMLs and NAWM. Furthermore, structural, diffusion, and physiologic parameters have not been simultaneously evaluated in WMLs versus NAWM to allow evaluation of correlations among MR imaging parameters, which might be redundant due to measurement of similar physiology or for technical reasons.

We hypothesized that even in the relatively healthy Coronary Artery Risk Development in Young Adults (CARDIA) cohort, 43-56 years of age, visible WMLs under-represent total white matter abnormality. Evidence of more widespread injury may formed consent to participate. The original cohort consisted of 5115 healthy black and white participants, $18-30$ years of age. ${ }^{22}$ In the 25th year of follow-up, at a mean of 50 years of age, brain MR imaging was performed between August 19, 2010, and August 31, 2011, in 719 randomly selected subjects in the CARDIA study who had no contraindications to MR imaging. Structural (T1, T2, FLAIR), diffusion (MD, FA), and physiologic data (arterial spinlabeling perfusion, breath-hold fMRI) were acquired, and complete, analyzable datasets were obtained in 463 subjects (On-line Fig 1) from 2 sites: the University of Minnesota $(n=250)$ and Kaiser-Permanente Division of Research $(n=213)$, both using 3T Tim Trio scanners (Siemens, Erlangen, Germany). Demographic composition of this subgroup is shown in the Table 1; these subjects are similar to the overall CARDIA 25th year of follow-up MR imaging cohort, ${ }^{1}$ and there was no significant difference on the basis of site except for body mass index (BMI) and smoking history.

\section{MR Imaging and Analysis}

This study was a retrospective analysis of prospectively acquired data, approved by the institutional review board of the University of Pennsylvania. The CARDIA brain MR imaging protocol has been published previously ${ }^{1}$; briefly, it included T1 (TR $=1900$ $\mathrm{ms}, \mathrm{TE}=2.89 \mathrm{~ms}, \mathrm{FOV}=250 \mathrm{~mm}$, thickness $=1 \mathrm{~mm}$, slices $=$ 176 , native resolution $=1 \mathrm{~mm}$ isotropic $), \mathrm{T} 2(\mathrm{TR}=3200 \mathrm{~ms}$, $\mathrm{TE}=409 \mathrm{~ms}, \mathrm{FOV}=250 \mathrm{~mm}$, thickness $=1 \mathrm{~mm}$, slices $=176$, native resolution $=1 \mathrm{~mm}$ isotropic), FLAIR $(\mathrm{TR}=6000 \mathrm{~ms}$, $\mathrm{TE}=285 \mathrm{~ms}, \mathrm{FOV}=258 \mathrm{~mm}$, thickness $=1 \mathrm{~mm}$, slices $=160$, native resolution $=1 \mathrm{~mm}$ isotropic), 30-direction diffusion tensor imaging $(\mathrm{TR}=7400 \mathrm{~ms}, \mathrm{TE}=82 \mathrm{~ms}, \mathrm{FOV}=246 \mathrm{~mm}$, thickness $=2.2 \mathrm{~mm}$, slices $=64$, native resolution $=2.2 \mathrm{~mm}$ isotropic), pseudocontinuous arterial spin-labeling perfusion $(\mathrm{TR}=4000 \mathrm{~ms}, \mathrm{TE}=11 \mathrm{~ms}, \mathrm{FOV}=220 \mathrm{~mm}$, thickness $=5 \mathrm{~mm}$, slices $=20$, native resolution $=3.4 \times 3.4 \times 6 \mathrm{~mm}$ ), and breathhold blood oxygen level-dependent (BOLD) fMRI (TR $=2000$ 


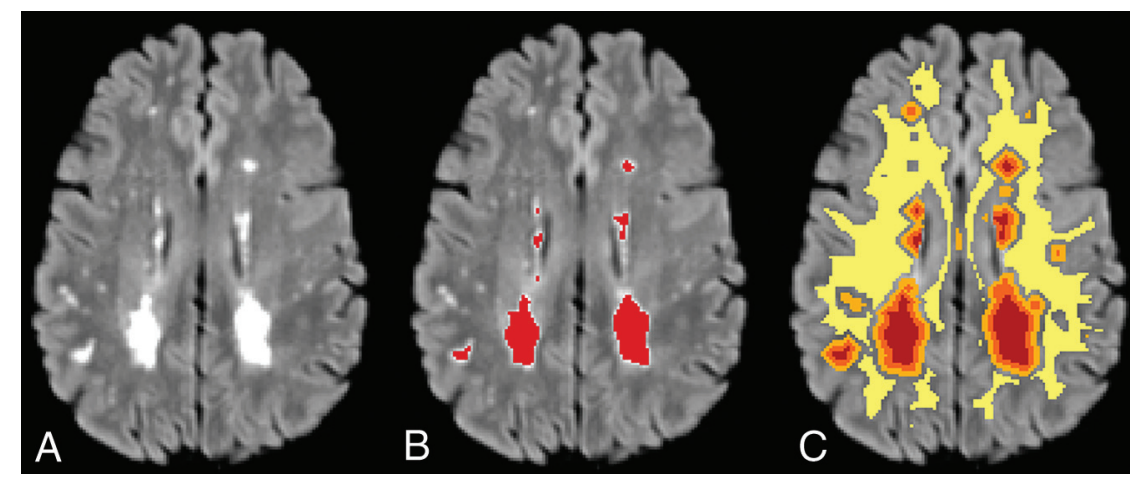

FIG 1. Sample FLAIR image showing WML (A); WML segmentation in red (B); and WML (red), $0-2$ $\mathrm{mm}$ (orange) and 2-4 mm (light orange) penumbra, and dNAWM (yellow) (C). dNAWM is eroded from GM to eliminate partial volume effects among these tissue types.

$\mathrm{ms}, \mathrm{TE}=25 \mathrm{~ms}, \mathrm{FOV}=224 \mathrm{~mm}$, thickness $=3.5 \mathrm{~mm}$, slices $=$ 35 , native resolution $=3.5 \mathrm{~mm}$ isotropic). Scanner performance was monitored with quarterly Alzheimer's Disease Neuroimaging Initiative and fBIRN phantom acquisitions (EZfMRI, Chicago, Illinois), with the scanners showing stability of phantom measurements throughout the study.

Processing of structural MR imaging sequences first involved histogram normalization and bias field correction of raw image data, which improve comparability of the uncalibrated, ordinal data, followed by template registration ${ }^{23}$ and semiautomated tissue segmentation. ${ }^{24}$ WML segmentation was performed using a previously validated supervised learning-based multimodal segmentation method. ${ }^{24,25}$ This support vector machine classifier was originally trained on multimodal MR imaging data from a separate training set with expert human manual segmentation of WMLs. It provides segmentation based on structural MR imaging (T1, T2, FLAIR) that strongly correlates with a definition of WMLs in the training set of a human observer. The model was applied on subjects in the CARDIA study to calculate binary WML masks and has been used in prior analyses of CARDIA imaging data. WML masks were morphologically dilated to define 2-mm rims of adjacent penumbral tissue $(0-2$ and $2-4 \mathrm{~mm}$ ) within tissue classified as NAWM. These 2-mm ROIs were selected on the basis of a compromise between the intrinsic spatial resolution of the acquired data and the relatively narrow expected transition from WMLs to normal tissue. Voxels within these penumbral ROIs are more likely to have been exposed to similar vascular injury compared with those in WMLs and therefore are more likely to be abnormal; however, there is no a priori standard for subclassifying these voxels. Distant normal-appearing white matter (dNAWM) was defined by eroding the white matter segmentation mask from the GM and WML/penumbra ROIs by $2 \mathrm{~mm}$ to avoid partial volume effects.

From DTI, we calculated FA and MD scalar maps. CBF maps were calculated from pseudocontinuous arterial spin-labeling. ${ }^{26}$ Maps of VR as measured by the percentage signal change in BOLD fMRI between breath-hold and rest states were generated using a method similar to that of Murphy et al. ${ }^{27}$ To summarize, breathholding was used to elevate blood $\mathrm{CO}_{2}$ and thereby induce cerebral vasodilation to effect changes in BOLD signal. During acquisition of BOLD fMRI, subjects were cued to follow four 16-second breathholds using E-Prime (Psychology Software Tools, Sharpsburg, Pennsylvania). Using FSL (http://www.fmrib.ox.ac.uk/fsl), we mo- tion-corrected the resulting images and smoothed them, and a whole-brain generalized linear model analysis was performed using a 9-second delay to account for hemodynamic lag. We excluded subjects without activation of the superior sagittal sinus, a marker of breath-hold compliance, measured as a $z$ score within the superior sagittal sinus of $<2.3$. VR $z$ score maps were thresholded at $z>2.3$, and percentage activation was calculated relative to the median $z$ score in the superior sagittal sinus. Only voxels with activation above this threshold were included for analysis. Maps for FA, MD, CBF, and VR were registered to $\mathrm{T} 1$ space and interpolated to $1-\mathrm{mm}$ isotropic resolution.

$3 \mathrm{D}$ volumes and imaging maps were aligned using FSL. In each of the 4 ROIs-WML, 0- to 2-mm penumbra, 2- to 4-mm penumbra, and dNAWM (Fig 1) - mean intensity was extracted from each of the acquired sequences, resulting in mean intensity values for each of the 4 regions for FLAIR, T1, T2, FA, MD, CBF, and VR. For VR, to evaluate the possible contributions of noise from subjects with few activated voxels, we repeated the analyses after exclusion of subjects with the lowest quartile of activated voxels for each ROI as a secondary analysis.

\section{Statistics}

Statistical analysis was performed using Python 2.7 (https:// www.python.org/download/releases/2.7/). Mean values for each parameter were compared between groups using Kruskal-Wallis and Wilcoxon signed rank pair-wise $t$ tests. Regression analysis of median intensity across regions was performed by applying a generalized estimating equation to $z$ score-normalized intensity measures. Correlation matrices using Pearson product-moment correlation coefficients were computed for intensities of each parameter. The Holm-Bonferroni correction was used to adjust for multiple comparisons in pair-wise $t$ tests and correlation matrices. Recursive feature elimination with stratified 10 -fold crossvalidation was performed to determine the optimal set of parameters that best predicted assignment to WML, 0- to 2-mm penumbra, 2- to 4-mm penumbra, and NAWM ROIs for each voxel. This method initially used all parameters to train the estimator; then, features with the lowest weights were recursively eliminated until further feature removal reduced classification accuracy. We investigated the associations of imaging parameters in WMLs and penumbras with clinical risk factors of systolic blood pressure, BMI, smoking status, and sedentary behavior, as previously defined, ${ }^{1}$ controlling for imaging site and demographic factors of age, sex, and race using ordinary least-squares linear regression modeling with $z$ score-transformed data, adjusting for age, sex, race, and site. Similarly, we evaluated overall cardiovascular risk using a derived risk factor score $\left(\mathrm{RF}_{\text {score }}\right.$ range, $\left.0-6\right)$ that was generated for each participant as a count of which of the following 6 risk factors were present: hypertension (systolic blood pressure $>140 \mathrm{~mm} \mathrm{Hg}$ or on antihypertensive medication), hypercholesterolemia (total serum cholesterol level $>100 \mathrm{mg} / \mathrm{dL}$ or 
Table 2: Regional volumes and mean intensity values for MRI parameters ${ }^{\mathrm{a}}$

\begin{tabular}{|c|c|c|c|c|c|}
\hline & WML & $\begin{array}{l}\text { Penumbra } \\
(0-2 \mathrm{~mm})\end{array}$ & $\begin{array}{c}\text { Penumbra } \\
(2-4 \mathrm{~mm})\end{array}$ & dNAWM & $\begin{array}{l}\text { Regression } \\
\text { Coefficient }\end{array}$ \\
\hline Mean volume $\left(\mathrm{cm}^{3}\right)$ & $\begin{array}{c}0.738(0.842) \\
{[0.661-0.815]}\end{array}$ & $\begin{array}{c}2.78(2.26) \\
{[2.57-2.99]}\end{array}$ & $\begin{array}{c}5.81(4.21) \\
{[5.43-6.19]}\end{array}$ & $\begin{array}{c}219(3.68) \\
{[186-253]}\end{array}$ & NA \\
\hline FLAIR intensity & $\begin{array}{c}136(10.1) \\
{[135-137]}\end{array}$ & $\begin{array}{c}98.9(3.50) \\
{[98.6-99.2]}\end{array}$ & $\begin{array}{c}79.4(3.71) \\
{[79.1-79.7]}\end{array}$ & $\begin{array}{l}70.8(2.58) \\
{[70.6-71.0]}\end{array}$ & -0.854 \\
\hline T1 intensity & $\begin{array}{l}116(6.43) \\
{[115-117]}\end{array}$ & $\begin{array}{l}132(5.80) \\
{[132-133]}\end{array}$ & $\begin{array}{l}142(4.50) \\
{[142-142]}\end{array}$ & $\begin{array}{c}156(1.31) \\
{[156-156]}\end{array}$ & 0.824 \\
\hline T2 intensity & $\begin{array}{l}115(8.62) \\
{[114-115]}\end{array}$ & $\begin{array}{c}88.3(6.82) \\
{[87.7-88.9]}\end{array}$ & $\begin{array}{c}74.1(4.75) \\
{[73.7-74.5]}\end{array}$ & $\begin{array}{c}60.6(1.02) \\
{[60.5-60.7]}\end{array}$ & -0.734 \\
\hline FA & $\begin{array}{l}0.279(0.0554) \\
{[0.273-0.284]}\end{array}$ & $\begin{array}{l}0.328(0.0411) \\
{[0.324-0.331]}\end{array}$ & $\begin{array}{l}0.374(0.0380) \\
{[0.371-0.377]}\end{array}$ & $\begin{array}{l}0.384(0.0254) \\
{[0.382-0.386]}\end{array}$ & 0.630 \\
\hline $\operatorname{MD}\left(10^{-3}\right)$ & $\begin{array}{l}3.68(0.662) \\
{[3.62-3.73]}\end{array}$ & $\begin{array}{l}3.23(0.445) \\
{[3.19-3.27]}\end{array}$ & $\begin{array}{l}2.97(0.344) \\
{[2.93-3.00]}\end{array}$ & $\begin{array}{c}2.33(0.112) \\
{[2.32-2.34]}\end{array}$ & -0.578 \\
\hline $\mathrm{CBF}(\mathrm{mL} / 100 \mathrm{~g} / \mathrm{min})$ & $\begin{array}{c}25.2(10.3) \\
{[24.3-26.1]}\end{array}$ & $\begin{array}{l}26.9(9.37) \\
{[26.1-27.7]}\end{array}$ & $\begin{array}{c}28.8(9.06) \\
{[28.0-29.6]}\end{array}$ & $\begin{array}{c}34.4(8.56) \\
{[33.6-35.2]}\end{array}$ & 0.306 \\
\hline $\begin{array}{l}\text { VR } \\
\text { (Mean \% change) }\end{array}$ & $\begin{array}{l}0.776(0.493) \\
{[0.731-0.821]}\end{array}$ & $\begin{array}{c}0.779(0.379) \\
{[0.745-0.813]}\end{array}$ & $\begin{array}{c}0.790(0.328) \\
{[0.760-0.820]}\end{array}$ & $\begin{array}{c}0.681(0.377) \\
{[0.647-0.715]}\end{array}$ & 0.192 \\
\hline
\end{tabular}

Note:-NA indicates not applicable.

a SD is in parentheses, and 95\% confidence intervals for intensity parameters are in brackets. Kruskal-Wallis tests were significant for all parameters, and pair-wise comparisons had $P<.001$ except that the WML and penumbral ROIs did not show significant differences in VR. The slope of linear regression across the 4 regions for intensity variables using a median value is shown in the last column; regression analyses were all statistically significant with $P<.001$.
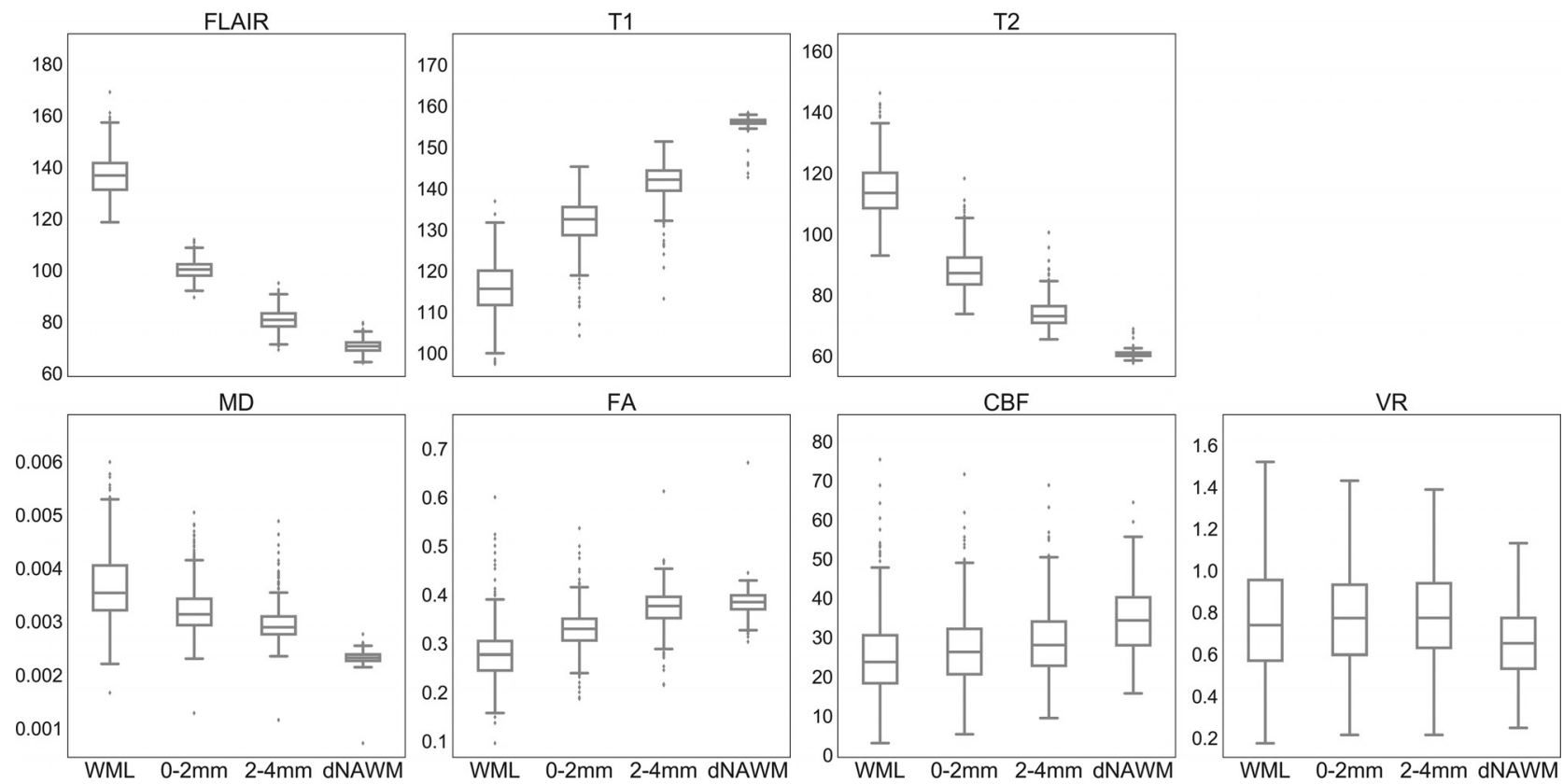

FIG 2. Boxplots of mean intensity values for each region. CBF is measured in cubic millimeters $/ 100 \mathrm{~g} / \mathrm{min}$, and VR shows the mean percentage signal change. For vascular reactivity, outliers are excluded (comprising $\sim 10 \%$ of the sample) to better demonstrate differences among means. A graph with outliers is available (On-line Fig 2). For each parameter, mean values are significantly different among all pair-wise comparisons between ROls with corrected $P<.001$, except for VR in which comparison of WML and dNAWM shows corrected $P=.001$, and there is no significant difference among WMLs and the 2 penumbra regions $(P>.4)$.

on cholesterol-lowering medication), sedentary behavior $(>75$ th percentile, equivalent to $>8.5$ hours of sedentary behavior per day), diabetes (if present), BMI (if $\geq 30$ ), or history of smoking (current and former smokers).

\section{RESULTS}

WML volumes averaged $0.738 \pm 0.842 \mathrm{~cm}^{3}$ (range, 0.005-7.27 $\mathrm{cm}^{3}$ ) in the 463 subjects from the CARDIA 25th year of follow-up cohort included in this study. Derived volumes of 0 - to 2-mm penumbra, 2- to 4-mm penumbra, and dNAWM measured at $2.78 \pm 2.26,5.81 \pm 4.21$, and $219 \pm 36.8 \mathrm{~cm}^{3}$, respectively.
We found significant differences for most parameters across ROIs (Table 2 and Fig 2). Mean FLAIR intensity, T2, and MD were higher in WMLs than in dNAWM, while mean T1 intensity, FA, and $\mathrm{CBF}$ were lower (all $P<.001$ ). For all these parameters, penumbral tissue showed intermediate values that were significantly different from each other and from both WMLs and dNAWM ( $P<.001$ for all pair-wise $t$ tests). Regression analysis by group demonstrated a strong effect of tissue type on signal intensities for FLAIR, T1, T2, MD, and FA, all statistically significant $(P<.001)$, with the strongest effects seen for structural parameters (Table 2); these correlations were not materially affected by adjusting for 


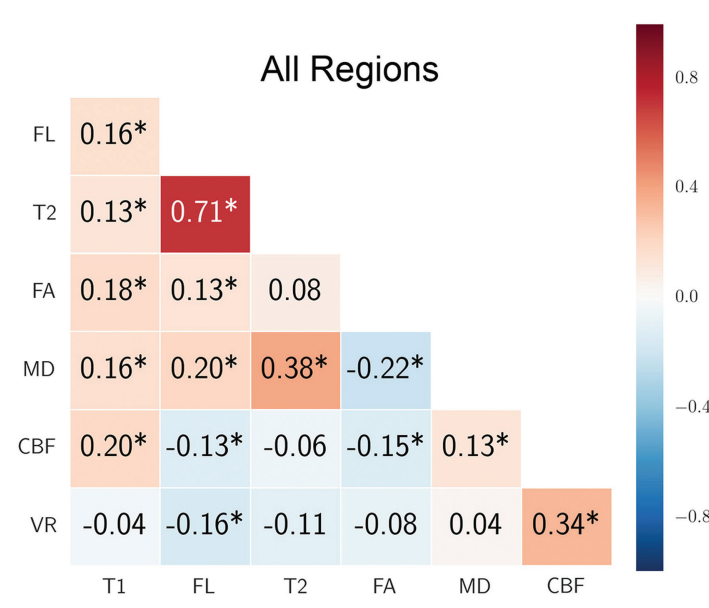

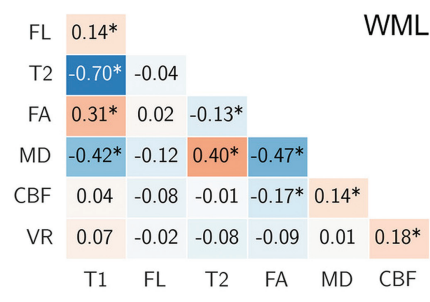

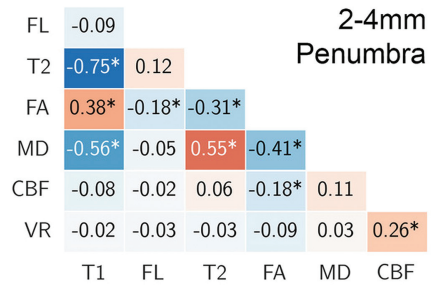

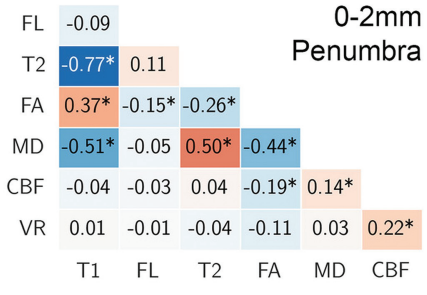

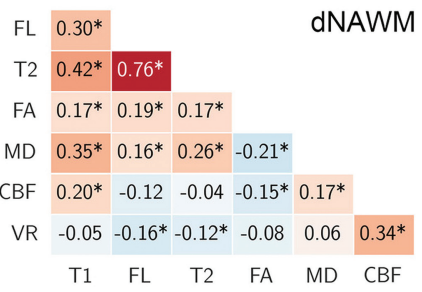

FIG 3. Correlations among the $7 \mathrm{MR}$ imaging variables (Pearson $r$ ). Correlation is shown for all voxels in all regions combined and separately for each region. Positive correlations are shaded red, and negative correlations are shaded blue. The asterisk indicates Bonferroni-Holm corrected $P<.05$.

site. There was no association between intensity values and subject age for any ROI (data not shown).

VR calculated from breath-hold fMRI showed a lower mean number of activated voxels in WM versus GM (67.0\% $\pm 27.9 \%$ versus $78.3 \% \pm 29.5 \%$ showed activation, respectively, $P<.001$ ). Activated voxels in the WM also showed a lower percentage signal change compared with GM $(0.865 \% \pm 0.40 \%$ versus $1.43 \% \pm$ $0.57 \%, P<.001)$. Compared with prior studies with $5 \% \mathrm{CO}_{2}$ inhalation, which achieves more reproducible blood $\mathrm{CO}_{2}$ levels, our VR results showed similar values of WM percentage activation $^{28}$ and ratios between GM and WM activation. ${ }^{29}$ However, the VR data are less reliable than other measures because values are not available for all voxels, especially in WMLs (On-line Table $1)$, and are more variable.

Compared with dNAWM, mean VR was significantly higher in WMLs $(P=.001)$ and penumbral regions $(P<.001$; Table 2 , Fig 2, and On-line Fig 2). Because only a subset of voxels within a region show measurable activation to contribute to VR signal, variability in mean VR measures was high in subjects with small lesion volumes, probably due to noise. Repeating the comparison but excluding 117 subjects in the lowest quartile of lesion volumes showed a decrease in mean WML VR from $0.78 \pm 0.49$ to $0.75 \pm$ 0.38 , with reduction in variability, but the relationships to the other regions remained similar.

We investigated the correlations among the 6 parameters to evaluate whether some of these variables provided redundant information (Fig 3). There were few consistent relationships among variables, and most correlations were low. MD showed the most stable correlations to other parameters across regions, but these correlations were moderate at best. Strong correlations were seen only with the positive correlation of T2 and FLAIR in dNAWM and across all regions combined and with a negative correlation between T1 and T2 in WMLs and penumbras; however, both of these relationships were weak or reversed in the other regions. CBF and VR were weakly correlated with other variables. Overall correlations were dominated by dNAWM, which had the largest volumes. The variability of magnitude and direction of the correlations among parameters suggests that these parameters provide unique information that may be useful for characterizing abnormal white matter tissue. To investigate which features are most useful for classification of voxels into the WML, penumbral, and NAWM ROIs, we used recursive feature elimination. ${ }^{30}$ This process determined that T1, FLAIR, and T2 provided optimal classification of voxels to these ROIs. MD was the next most relevant but was not selected, followed by FA, CBF, and VR. On-line Fig 3 shows the cross-validation accuracy versus the number of features selected.

Finally, we evaluated the relationships between vascular risk factors and signal intensities for FLAIR and FA, as representative structural and DTI measures, as well as CBF and VR in WMLs and penumbral ROIs. As expected, we found significant associations of higher WML volume with higher systolic blood pressure $(P<$ $.001)$, even without correcting for antihypertensive therapy, and with the $\mathrm{RF}_{\text {score }}(P<.001)$. In multivariate modeling of regional intensities adjusting for age, race, sex, and site, the $\mathrm{RF}_{\text {score }}$ showed significant association only with mean CBF in the WML and both penumbral ROIs (On-line Table 2), with a higher $\mathrm{RF}_{\text {score }}$ associated with lower mean CBF. Next, we looked at a model with the same demographic factors but including hypertension, hypercholesterolemia, smoking history, diabetes, sedentary behavior, and BMI as independent variables, instead of the risk score (On-line Table 3). Sedentary behavior was associated with significant differences in mean $\mathrm{T} 1$ and $\mathrm{T} 2$ within WMLs and with VR in the 2- to 4-mm penumbra. Smoking history was associated with significant differences in CBF and VR within lesions and with T1 and MD in both penumbras. BMI was inversely associated with CBF across all 3 regions. Other risk factors did not show significant associations in these models.

\section{DISCUSSION}

While WMLs usually appear as discrete lesions, prior studies in elderly populations have found associations with abnormality in other, visually normal WM tissue. Although strongly associated with age and hypertension, WMLs are seen in young, normotensive individuals. We examined MR imaging characteristics in WMLs and penumbra in adults $43-56$ years of age in the CARDIA 
study. The CARDIA Brain MRI cohort has a much lower mean age and WML volume compared with most other studies that have evaluated WMLs and penumbra; for comparison, the study of de Groot et $\mathrm{al}^{7}$ had a mean age of 67 years with a median WML volume of $3.4 \mathrm{~cm}^{3}$. The Women's Health Initiative Memory Study, which used the same WML classifier method as in the current study, had a mean age of 78.5 years and mean WML volume of $4.3 \mathrm{~cm}^{3}$, 31 and the study of Promjunyakul et $\mathrm{al}^{16}$ had a mean age of 85 years, with a mean WML volume of $11.2 \mathrm{~cm}^{3}$. Most CARDIA MRI participants were normotensive (57\%), with $11 \%$ prehypertensive and $32 \%$ hypertensive. ${ }^{1}$

Despite the low lesion burden, we found statistically significant abnormalities in all measured structural, diffusion, and physiologic MR imaging parameters in penumbral tissue, encompassing, on average, more than 10-fold larger volume than WMLs (Fig 2 and Table 2). Signal abnormalities in WML and penumbral regions were worsened when vascular risk factors were present, specifically with relationships between systolic blood pressure and FLAIR intensity and between systolic blood pressure and BMI and CBF. These findings indicate that as in the elderly, WM damage is more extensive in middle age than represented by the typical low WML volume, suggesting that early intervention for reversible cardiovascular risk factors may yield benefit in slowing progression of this damage. Recent results from the Systolic Blood Pressure Intervention Trial (SPRINT) have shown that aggressive blood pressure control may slow progression of WML volume, ${ }^{32}$ and it is possible that such an intervention would also affect penumbral tissue. Most interesting, we did not find an association between regional signal intensities and age, which has been shown to be the strongest risk factor for WMLs in prior studies. It is possible that at the younger age of this cohort, lesions are primarily the consequence of hypertension; therefore, growth could potentially be prevented with adequate antihypertensive therapy. Overall, we found limited associations between cardiovascular risk factors and regional intensity values; however, effects were shown in multivariate analyses between a cumulative risk factor score and, individually, with sedentary behavior, smoking history, and BMI. These associations require further investigation, particularly as the exposure lengthens with aging.

The physiologic MR imaging variables, CBF and VR, have been less extensively studied in WMLs and penumbra. Our study confirms prior reports showing decreased CBF in WMLs ${ }^{10,11}$ and penumbra compared with NAWM. ${ }^{16}$ Unlike the other imaging parameters, which probably measure sequelae from injury, $\mathrm{CBF}$ and VR may more directly measure etiologic abnormalities for WMLs; most interesting, CBF was the only variable significantly associated with the number of cardiovascular risk factors. Regional differences were less pronounced in the physiologic $\mathrm{CBF}$ and VR data, partially related to lower signal to noise and resolution and greater biologic variability. Compared with VR, CBF is more technologically mature with greater potential for widespread implementation.

The few studies that have evaluated VR in WMLs mostly evaluated global VR, which is dominated by GM, and more often used transcranial Doppler sonography rather than MR imaging. Hypertension and vascular risk factors have been associated with decreased VR; some but not all studies showed adverse associations of global cerebral VR with WMLs. ${ }^{33-35}$ Studies in elderly subjects measuring BOLD VR with a more reproducible $\mathrm{CO}_{2}$ inhalation method showed a 50\%-60\% decreased VR in WML versus NAWM. ${ }^{12,28}$ In our study, the mean VR was unexpectedly higher in WMLs and penumbra than in ANAWM; if validated, this finding might suggest a difference in physiology in WMLs and at-risk tissue in younger or subjects with low-WML volume compared with elderly or subjects with high-WML volume. Increased VR may indicate a compensatory, possibly protective, vasoreactivity versus an indication of intrinsic, possibly harmful, abnormality of the vessels.

The mechanisms behind WML propagation are unknown. Vascular compromise may spread from extant WMLs, or independent events may accumulate in vulnerable territory. We found that adjacent tissue, including that 2-4 $\mathrm{mm}$ removed from WMLs, showed detectable abnormalities on structural imaging, measures of white matter integrity, and physiologic parameters. For all parameters except VR, penumbra showed intermediate values transitioning between WMLs and dNAWM, suggesting lesser injury. Longitudinal evaluation comparing regions that develop into lesions versus those that do not will likely identify heterogeneity within penumbral tissue and show whether these parameters can predict lesion growth. Unfortunately, prospective prediction of lesion development has not yet been successful. Scoring each voxel based on a combination of MR imaging parameters may provide a means to identify at-risk regions. We found that a combination of FLAIR, T1, and T2 provided optimal, nonredundant classification of voxels into the WML, penumbral, and dNAWM ROIs; however, it remains to be seen whether longitudinal change in WMLs is better predicted by baseline structural or physiologic measures or a combination.

There are several limitations of this study. Due to lower resolution of diffusion and functional sequences compared with structural imaging, partial volume effects likely affect calculation of mean intensities. However, significant differences were seen between WMLs and the 2- to 4-mm penumbra, which would be less affected by partial volume effects from WMLs. The WML segmentation classifier used to identify WMLs is conservative, and as a result, some tiny WMLs may be included in other regions, which should decrease statistical differences by increasing variability. Using two, 2-mm concentric penumbral rings was a compromise between the need to evaluate a relatively narrow band of tissue around WMLs and the need to reduce partial volume effects. Others have shown similar gradated changes extending even further around WMLs. ${ }^{16}$ The morphologic dilation used to generate the penumbral ROI overlooks the expected heterogeneity of penumbral tissue; however, mixing tissues with varying levels of injury in concentric ROIs is expected to decrease the power of this study.

T1, T2, and FLAIR intensities vary depending on scanner and imaging parameters. Our data were acquired on the same scanner model with the same imaging parameters, and normalization techniques were implemented to harmonize the data; however, these features affect generalizability to other sites. We made no correction for the mild, normal regional variations of signal intensity in the brain, which has been previously performed. ${ }^{7}$ Nor- 
mal signal variations may accentuate some regional differences; for example, FLAIR is normally slightly increased in periventricular regions most commonly affected by WMLs relative to NAWM. FA, however, shows significant decreases in regions that normally have higher FA, suggesting that applying regional intensity corrections might result in even greater differences.

\section{CONCLUSIONS}

Even in relatively healthy, middle-aged adults with low total WML burden, WMLs have a substantial penumbra of tissue with abnormalities quantifiable by structural and physiologic MR imaging parameters, in some individuals involving volumes $>10$ times larger than WMLs visible on FLAIR. Furthermore, the gradation of signal abnormalities in penumbral tissue suggests early/ milder injury, compatible with the hypothesis that injury expands from established lesions; this penumbral tissue may be a target for therapeutic intervention to prevent worsening of WMLs. Multimodal MR imaging may better define injured white matter than methods that use only structural data. Further investigation is needed to determine the optimal combination of parameters to prospectively identify the full extent of abnormal white matter and the effects of these abnormalities on neural circuits and cognition. An epidemiologic priority is to examine whether the volume and characteristics of these expanded injury regions predict future lesions and risk of brain-related disease.

\section{ACKNOWLEDGMENTS}

The authors thank Raymond Pomponio for statistical assistance.

Disclosures: Ilya M. Nasrallah—RELATED: Grant: National Institutes of Health*; Support for Travel to Meetings for the Study or Other Purposes: National Institutes of Health*; UNRELATED: Grants/Grants Pending: National Institutes of Health.* Guray Erus-RELATED: Grant: National Institutes of Health.* John A. Detre-RELATED: Grant: National Institutes of Health*; UNRELATED: Consultancy: Blackfynn, CHDI Foundation, Dana Foundation, National Institutes of Health, Comments: I have provided expert advice on neuroimaging to the University of Pennsylvania spinoff startup company Blackfynn. I have received stock options. I have provided consulting to the CHDI Foundation, a private foundation involved in Huntington disease research, but none in the last year. I am compensated as a grant reviewer for the Dana Foundation and the National Institutes of Health, total approximately $\$ 2000$ per year; Grants/Grants Pending: National Institutes of Health*; Payment for Manuscript Preparation: Dana Foundation, Comments: I received $\$ 10,000$ from the Dana Foundation for assisting with a Web article on neuroimaging; Patents (Planned, Pending or (ssued): University of Pennsylvania, Comments: I am listed as a coinventor on 2 University of Pennsylvania patents for optical monitoring of cerebral blood flow. Neither has been licensed. Lenore J. Launer-UNRELATED: Employment: National Institutes of Health. Robert N. Bryan—RELATED: Grant: National Institutes of Health*; UNRELATED: Board Membership: Galileo CDS, Comments: no cash transaction or value; Grants/Grants Pending: National Institutes of Health; Patents (Planned, Pending or Issued): University of Pennsylvania patent license to Galileo CDS; Stock/Stock Options: Galileo CDS. *Money paid to the institution.

\section{REFERENCES}

1. Launer LJ, Lewis CE, Schreiner PJ, et al. Vascular factors and multiple measures of early brain health: CARDIA Brain MRI Study. PLoS One 2015;10:e0122138 CrossRef Medline

2. Jokinen H, Kalska H, Ylikoski R, et al; LADIS group. Longitudinal cognitive decline in subcortical ischemic vascular disease: the LADIS study. Cerebrovasc Dis 2009;27:384-91 CrossRef Medline

3. Verdelho A, Madureira S, Moleiro C, et al; LADIS Study. White matter changes and diabetes predict cognitive decline in the elderly: the LADIS study. Neurology 2010;75:160-67 CrossRef Medline

4. Debette S, Markus HS. The clinical importance of white matter hy- perintensities on brain magnetic resonance imaging: systematic review and meta-analysis. BMJ 2010;341:c3666 CrossRef Medline

5. Teodorczuk A, Firbank MJ, Pantoni L, et al; LADIS Group. Relationship between baseline white-matter changes and development of late-life depressive symptoms: 3-year results from the LADIS study. Psychol Med 2010;40:603-10 CrossRef Medline

6. Inzitari D, Pracucci G, Poggesi A, et al; LADIS Study Group. Changes in white matter as determinant of global functional decline in older independent outpatients: three-year follow-up of LADIS (leukoaraiosis and disability) study cohort. BMJ 2009;339:b2477 CrossRef Medline

7. de Groot M, Verhaaren BF, de Boer R, et al. Changes in normalappearing white matter precede development of white matter lesions. Stroke 2013;44:1037-42 CrossRef Medline

8. Brown WR, Thore CR. Review: cerebral microvascular pathology in ageing and neurodegeneration. Neuropathol Appl Neurobiol 2011; 37:56-74 CrossRef Medline

9. Fazekas F, Kleinert R, Offenbacher H, et al. Pathologic correlates of incidental MRI white matter signal hyperintensities. Neurology 1993;43:1683-89 CrossRef Medline

10. O'Sullivan M, Lythgoe DJ, Pereira AC, et al. Patterns of cerebral blood flow reduction in patients with ischemic leukoaraiosis. $\mathrm{Neu}$ rology 2002;59:321-26 CrossRef Medline

11. Brickman AM, Zahra A, Muraskin J, et al. Reduction in cerebral blood flow in areas appearing as white matter hyperintensities on magnetic resonance imaging. Psychiatry Res 2009;172:117-20 CrossRef Medline

12. Sam K, Crawley AP, Poublanc J, et al. Vascular dysfunction in leukoaraiosis. AJNR Am J Neuroradiol 2016;37:2258-64 CrossRef Medline

13. Topakian R, Barrick TR, Howe FA, et al. Blood-brain barrier permeability is increased in normal-appearing white matter in patients with lacunar stroke and leucoaraiosis. J Neurol Neurosurg Psychiatry 2010;81:192-97 CrossRef Medline

14. Grueter BE, Schulz UG. Age-related cerebral white matter disease (leukoaraiosis): a review. Postgrad Med J 2012;88:79-87 CrossRef Medline

15. Conklin J, Silver FL, Mikulis DJ, et al. Are acute infarcts the cause of leukoaraiosis? Brain mapping for $\mathbf{1 6}$ consecutive weeks. Ann Neurol 2014;76:899-904 CrossRef Medline

16. Promjunyakul N, Lahna D, Kaye JA, et al. Characterizing the white matter hyperintensity penumbra with cerebral blood flow measures. Neuroimage Clin 2015;8:224-29 CrossRef Medline

17. Maniega SM, Valdés Hernandez MC, Clayden JD, et al. White matter hyperintensities and normal-appearing white matter integrity in the aging brain. Neurobiol Aging 2015;36:909-18 CrossRef Medline

18. Bernbaum M, Menon BK, Fick G, et al. Reduced blood flow in normal white matter predicts development of leukoaraiosis. J Cereb Blood Flow Metab 2015;35:1610-15 CrossRef Medline

19. Schmidt R, Ropele S, Ferro J, et al; LADIS study group. Diffusionweighted imaging and cognition in the leukoariosis and disability in the elderly study. Stroke 2010;41:e402-08 CrossRef Medline

20. Pelletier A, Periot O, Dilharreguy B, et al. Age-related modifications of diffusion tensor imaging parameters and white matter hyperintensities as inter-dependent processes. Front Aging Neurosci 2016;7: 255 CrossRef Medline

21. Maillard P, Carmichael O, Harvey D, et al. FLAIR and diffusion MRI signals are independent predictors of white matter hyperintensities. AJNR Am J Neuroradiol 2013;34:54-61 CrossRef Medline

22. Friedman GD, Cutter GR, Donahue RP, et al. CARDIA: study design, recruitment, and some characteristics of the examined subjects. J Clin Epidemiol 1988;41:1105-16 CrossRef Medline

23. Shen D, Davatzikos C. HAMMER: hierarchical attribute matching mechanism for elastic registration. IEEE Trans Med Imaging 2002; 21:1421-39 CrossRef Medline

24. Zacharaki EI, Kanterakis S, Bryan RN, et al. Measuring brain lesion progression with a supervised tissue classification system. Med Image Comput Comput Assist Interv 2008;11:620-27 Medline 
25. Lao Z, Shen D, Liu D, et al. Computer-assisted segmentation of white matter lesions in 3D MR images using support vector machine. Acad Radiol 2008;15:300-13 CrossRef Medline

26. Wang Z, Aguirre GK, Rao H, et al. Empirical optimization of ASL data analysis using an ASL data processing toolbox: ASLtbx. Magn Reson Imaging 2008;26:261-69 CrossRef Medline

27. Murphy K, Harris AD, Wise RG. Robustly measuring vascular reactivity differences with breath-hold: normalising stimulus-evoked and resting state BOLD fMRI data. Neuroimage 2011;54:369-79 CrossRef Medline

28. Uh J, Yezhuvath U, Cheng Y, et al. In vivo vascular hallmarks of diffuse leukoaraiosis. J Magn Reson Imaging 2010;32:184-90 CrossRef Medline

29. Reich T, Rusinek H. Cerebral cortical and white matter reactivity to carbon dioxide. Stroke 1989;20:453-57 CrossRef Medline

30. Guyon I, Weston J, Barnhill S, et al. Gene selection for cancer classification using support vector machines. Machine Learning 2002;46: 389-422 CrossRef

31. Kuller LH, Margolis KL, Gaussoin SA, et al; Women's Health Initia- tive Memory Study Research Group. Relationship of hypertension, blood pressure, and blood pressure control with white matter abnormalities in the Women's Health Initiative Memory Study (WHIMS)-MRI trial. J Clin Hypertens (Greenwich) 2010;12:203-12 CrossRef Medline

32. Nasrallah IM; Alzheimer's Association International Conference. A Randomized Trial of Intensive Versus Standard Systolic Blood Pressure Control and Brain Structure: SPRINT MIND MRI. Chicago, IL; July 25, 2018

33. Matsushita K, Kuriyama Y, Nagatsuka K, et al. Periventricular white matter lucency and cerebral blood flow autoregulation in hypertensive patients. Hypertension 1994;23:565-68 CrossRef Medline

34. Bakker SL, de Leeuw FE, de Groot JC, et al. Cerebral vasomotor reactivity and cerebral white matter lesions in the elderly. Neurology 1999;52:578 -83 CrossRef Medline

35. Birns J, Jarosz J, Markus HS, et al. Cerebrovascular reactivity and dynamic autoregulation in ischaemic subcortical white matter disease. J Neurol Neurosurg Psychiatry 2009;80:1093-98 CrossRef Medline 